krebs HR+/HER2- eingesetzt, der unter endokriner Therapie (ET) progredierte [Sledge GW et al. ASCO. 2017;Abstr 1000]. Wie bereits von den CDK4/6-Inhibitoren Palbociclib und Ribociclib bekannt, soll hier die endokrine Resistenz durchbrochen und das Ansprechen auf die ET verbessert werden. Das PFS stieg mit Abemaciclib gegenüber Fulvestrant plus Placebo (16 vs. 9 Monate, HR 0,553). Ähnliche Daten zeigte auch die PALOMA-3-Studie [Cristofanilli M et al. Lancet Oncol. 2016;17(4): 425-39]. Neben der bekannten Nebenwirkung Neutropenie trat unter Abemaciclib häufiger Diarrhö auf als unter anderen CDK4/6-Inhibitoren. In Zukunft werden also, nach wahrscheinlich erfolgter Zulassung, drei CDK4/6-Inhibitoren verfügbar sein.

In der Phase-IIIb-Sicherheits-Studie KAMILLA wurde der Einsatz von Trastuzumab Emtansin (T-DM1) beim vorbehandelten, fortgeschrittenen, HER2positiven Mammakarzinom überprüft [Barrios CH et al. ASCO. 2017;Abstr 1033]. Der Wirkstoff war insgesamt gut verträglich. Je weniger Vortherapien eingesetzt worden waren, desto besser war das Ansprechen. Die Ergebnisse zur Sicherheit der Substanz waren mit denen bekannter Vorstudien vergleichbar.

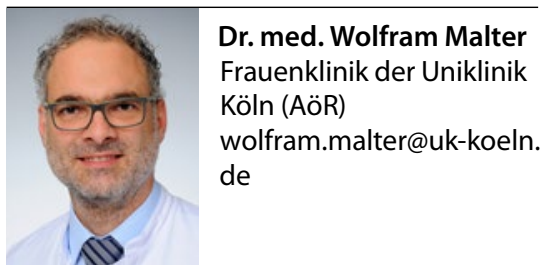

\title{
Neue Daten zur NSCLC-Therapie
}

Beim Lungenkarzinom standen insbesondere Verbesserungen zur Therapie des nichtkleinzelligen Lungenkarzinoms (NSCLC) im Vordergrund.

Eine zielgerichtete Therapie wird auch bei Tumoren mit seltenen molekularen Alterationen empfohlen, wie gezeigt werden konnte bei NSCLC-Patienten mit BRAF-V600-Mutation [Subbiah V et al. ASCO. 2017;Abstr 9074] oder MET-Exon14-Mutation [Awad et al. ASCO. 2017;Abstr 8511]. Bei anderen molekularen Alterationen (z. B. HER2) ist das Ausmaß des Nutzens einer zielgerichteten Therapie noch nicht eindeutig geklärt. Eine breitere molekulare Testung ist sinnvoll. Eindrucksvoll konnte die Wirksamkeit von Tyrosinkinaseinhibitoren (TKI) der nächsten Generation in einer globalen Phase-III-Studie gezeigt werden. 303 bis- her unbehandelte Patienten mit ALK-aktiviertem NSCLC erhielten entweder Alectinib oder Crizotinib. Nach einer medianen Nachbeobachtungszeit von 18,6 Monaten war das progressionsfreie Überleben (PFS, primärer Endpunkt), in der Alectinib-Gruppe signifikant besser ( $\mathrm{Ha}$ zard Ratio [HR] 0,47, p < 0,0001) [Shaw AT et al. ASCO. 2017;LBA9008]. Zudem waren unter Alectinib die Ansprechrate höher (82,9 vs. $75,5 \%)$ und die höhergradigen Nebenwirkungen geringer (41 vs. $50 \%$ ). Patienten mit ZNS-Metastasen hatten unter Alectinib ebenso ein längeres PFS (HR 0,40) bei besserem zerebralen Ansprechen (81 vs. 50\%).

Auch der immuntherapeutische Ansatz wird weiterentwickelt: Explorative Daten der Phase-III-Studie OAK zeigten, dass eine Behandlung mit dem PD-L1-
Hemmer Atezolizumab über den Progress hinaus bei klinisch selektionierten Patienten zu einem Nutzen führen kann - mit einer erneuten Ansprechrate von $7,7 \%$, einem PFS von 1,7 Monaten und einer medianen Überlebenszeit von 12,7 Monaten [Gandara DR et al. ASCO. 2017; Abstr 9001]. In mehreren PhaseIB-Studien war das Nebenwirkungsprofil einer Erstlinientherapie aus platinhaltiger Kombinationschemotherapie und einem PD-1- oder PD-L1-Antikörper klinisch beherrschbar. Dieser vielversprechende Ansatz wird derzeit in Phase-III-Studien weiter verfolgt.

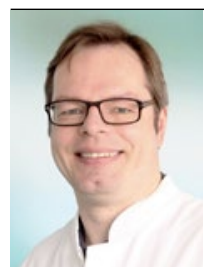

PD Dr. med. Niels Reinmuth Asklepios Fachkliniken München-Gauting Thorakale Onkologie n.reinmuth@asklepios. com hoch effektiv [Papadopoulos KP et al. ASCO. 2017; Abstr 9503]. Die Ansprechrate lag bei $46,2 \%$, die Tumorkontrollrate bei $69,2 \%$. Katharina Arnheim

Ublituximab, eine neue Option bei Hochrisiko-CLL In der Phase-III-Studie GENUINE führte die Addition von Ublitu- ximab zu Ibrutinib bei Patienten mit chronischer lymphatischer Leukämie (CLL) mit hohem Risiko zu einem signifikant besseren Ansprechen als eine Ibrutinib-Monotherapie, ohne die Toxizität klinisch relevant zu verstärken [Sharman JP et al. ASCO. 2017;Abstr 7504]. Ublituximab ist ein neuer monoklonaler Antikörper, der sich gegen ein spezi- fisches Epitop des CD20-Antigens richtet. Nachdem CLL-Patienten mit hohem Risiko von der Ibrutinib-Monotherapie weniger profitieren, sollte die Addition von Ublituximab die antikörperabhängige zellvermittelte Toxizität (ADCC) verbessern. Insgesamt waren 117 CLL-Patienten mit hohem Risiko (del[17p], del[11q], und/oder
TP53-Mutation) behandelt worden. Nach einem medianen Follow-up von 12 Monaten lag die Gesamtansprechrate bei $80 \%$ unter der Kombinationstherapie gegenüber $47 \%$ im lbrutinibMonotherapiearm ( $p<0,001)$. Zudem sprachen die Patienten schneller auf die Therapie an (median 1,97 vs. 3,8 Monate). Doris Berger 